\title{
Can we protect the kidneys after hypertensive pregnancy?
}

\author{
Julia J. Spaan MD PhD, Mark A. Brown MD
}

See related research article by Wang and colleagues on page 207 and at www.cmaj.ca/lookup/doi/10.1503/cmaj.120230

$\mathrm{T}$ here has been a worldwide increase in chronic kidney disease, which is associated with major health burdens. ${ }^{1}$ Clinicians are familiar with the progression of chronic kidney disease to end-stage renal disease requiring dialysis or transplantation, but the greater burden on patients with end-stage renal disease is their risk of cardiovascular disease. Not surprisingly, there is a major push to reduce the incidence of chronic kidney disease; this requires both the recognition of risk factors and appropriate screening at regular intervals. Several risk factors are commonly recognized, including age, hypertension, diabetes, ethnic background, smoking status, obesity and a family history of kidney disease. Hypertensive pregnancy has not traditionally been included in this list.

The study by Wang and colleagues adds new evidence to the discussion of maternal health after hypertensive pregnancy. ${ }^{2}$ It not only confirms the important finding of an increased risk of end-stage renal disease that was reported in a cohort study involving a Norwegian population, ${ }^{3}$ but it also confirms that a large component of this risk is the emergence of hypertension and diabetes in these women. This study was performed in Taiwan, an interesting country to study in this context because it has a very high prevalence of end-stage renal disease. ${ }^{1}$

Wang and colleagues ${ }^{2}$ used insurance claims data from over 26000 women with de novo hypertension during pregnancy and found about a 12 -fold increased risk of end-stage renal disease compared with women who did not have hypertension during pregnancy. Even the mild disorder of gestational hypertension without preeclampsia was associated with a 10-fold increased risk of end-stage renal disease; preeclampsia was associated with an even higher risk. This is important because about $10 \%$ of all pregnancies are complicated by one of these hypertensive disorders. ${ }^{2}$

Whether the findings from this Taiwanese population can be applied to the Canadian population is unclear. However, the fact that an association between hypertensive pregnancy and end-stage renal disease has been observed in 2 ethnic groups increases the generalizability.,3
Some perspective is necessary: 121 of 26651 $(0.45 \%)$ women developed end-stage renal disease over an average of 6.3 years of follow-up time, meaning that almost 1 in 200 women with hypertension during pregnancy needed dialysis in a relatively short period. Anecdotally, this is not what most renal physicians would see in a relatively young group of women. The authors acknowledge that many of these women may have had previously undetected mild chronic kidney disease and that there may be genetic influences. The bigger risk was for later diabetes $(4.4 \%)$ and hypertension (3.7\%), which have major long-term health implications.

This study adds to our knowledge of the mechanisms that may contribute to the development of end-stage renal disease after pregnancy. It clearly shows the importance of hypertension as a risk factor for end-stage renal disease, both for women with and without a history of a hypertensive disorder during pregnancy. Bellamy and colleagues reported that $25 \%$ of women with a pregnancy complicated by preeclampsia developed chronic hypertension within 10-20 years, compared with $4 \%$ after a normotensive pregnancy. ${ }^{4}$ The prevalence of hypertension $(0.3 \%)$ in this Taiwanese population of women studied by Wang and colleagues was lower than in most westernized countries, but this study still highlights the magnitude of the effect of hypertensive pregnancy on increasing the risk of chronic hypertension (to $3.7 \%$ ). ${ }^{2}$ Diabetes mellitus is well known to cause target-organ damage in the kidneys, resulting initially in microalbuminuria. As

\section{KEY POINTS}

- There is an increased risk of end-stage renal disease among women with a history of preeclampsia; those with the more benign disorder of gestational hypertension alone are also at increased risk.

- The development of hypertension, diabetes or both after pregnancy is an important mediator of chronic kidney disease.

- Blood pressure should be monitored regularly after a hypertensive disorder during pregnancy.

- Cardiovascular risk management in a structured multidisciplinary approach may reduce cardiovascular and renal disease after a hypertensive disorder during pregnancy. 
expected, women with diabetes were at increased risk of end-stage renal disease, but diabetes did not appreciably influence the associations between hypertensive disorder of pregnancy and end-stage renal disease. ${ }^{2}$

Some important points for clinical practice can be drawn from this study. Hypertension is a modifiable factor that can be addressed by lifestyle or pharmacologic interventions, and it is likely that good blood pressure control will reduce the risk of chronic kidney disease after a hypertensive disorder during pregnancy. Monitoring blood pressure after a hypertensive disorder during pregnancy has frequently been inadequate, missing half of the cases of chronic hypertension in an observational study. ${ }^{5}$ We have found that women who had a hypertensive pregnancy, compared with women who had a normotensive pregnancy, had higher blood pressure when assessed by 24-hour ambulatory blood pressure monitoring; they also had a higher body mass index and a greater tendency toward insulin resistance, but these differences were subtle and can be hard to detect. ${ }^{6}$

Women who have had a hypertensive disorder during pregnancy not only have an increased risk of chronic kidney disease, but they also have a doubled risk of cardiovascular diseases (e.g., stroke, cardiac ischemic events, deep venous thrombosis), as summarized in 2 meta-analyses. ${ }^{4,7}$ Of note, preeclampsia has recently been added as a major risk factor for cardiovascular disease by the American Heart Association. ${ }^{8}$ Ideally, cardiovascular risk management would follow a few months after a hypertensive pregnancy in a multidisciplinary approach, including general practitioners, renal and internal medicine specialists, cardiologists, obstetricians, dietary and physical therapists. ${ }^{9}$ A prominent role should be given to lifestyle interventions to promote a healthy lifestyle in these young families.

This study by Wang and colleagues ${ }^{2}$ is timely because it shows the importance of hypertension during pregnancy as a marker for future chronic kidney disease, cardiovascular disease and diabetes. It also highlights one of the current pitfalls of clinical practice: although these women have great attention paid to their high blood pressure during pregnancy, there is no structured follow-up of blood pressure or cardiovascular and renal risk factors after pregnancy. Better surveillance after pregnancy should help prevent not only chronic kidney disease but also cardiovascular disease.

\section{References}

1. Collins AJ, Foley RN, Herzog C, et al. Excerpts from the US Renal Data System 2009 Annual Data Report. Am J Kidney Dis 2010;55(1 Suppl 1):S1-420, A6-7.

2. Wang I-K, Muo C-H, Chang Y-C, et al. Association between hypertensive disorders during pregnancy and end-stage renal disease: a population-based study. CMAJ 2013;185:207-13.

3. Vikse BE, Irgens LM, Leivestad T, et al. Preeclampsia and the risk of end-stage renal disease. N Engl J Med 2008;359:800-9.

4. Bellamy L, Casas JP, Hingorani AD, et al. Pre-eclampsia and risk of cardiovascular disease and cancer in later life: systematic review and meta-analysis. BMJ 2007;335:974.

5. Callaway LK, David McIntyre H, Williams GM, et al. Diagnosis and treatment of hypertension 21 years after a hypertensive disorder of pregnancy. Aust N Z J Obstet Gynaecol 2011;51:437-40.

6. Mangos GJ, Spaan JJ, Pirabhahar S, et al. Markers of cardiovascular disease risk after hypertension in pregnancy. $J$ Hypertens 2012;30:351-8.

7. McDonald SD, Malinowski A, Zhou Q, et al. Cardiovascular sequelae of preeclampsia/eclampsia: a systematic review and meta-analyses. Am Heart J 2008;156:918-30.

8. Mosca L, Benjamin EJ, Berra K, et al. Effectiveness-based guidelines for the prevention of cardiovascular disease in women - 2011 update: a guideline from the American Heart Association. Circulation 2011;123:1243-62

9. Spaan J, Peeters L, Spaanderman M, et al. Cardiovascular risk management after a hypertensive disorder of pregnancy. Hypertension 2012;60:1368-73.

Affiliations: From the Department of Obstetrics and Gynecology and the GROW Research Institute (Spaan), Faculty of Health, Medicine and Life Sciences, Maastricht University Medical Center, Maastricht, the Netherlands; and the Department of Renal Medicine (Brown), St. George Hospital, University of New South Wales, Kogarah, New South Wales, Australia.

Contributors: Both of the authors contributed to the writing and editing of the manuscript and approved the final version submitted for publication.

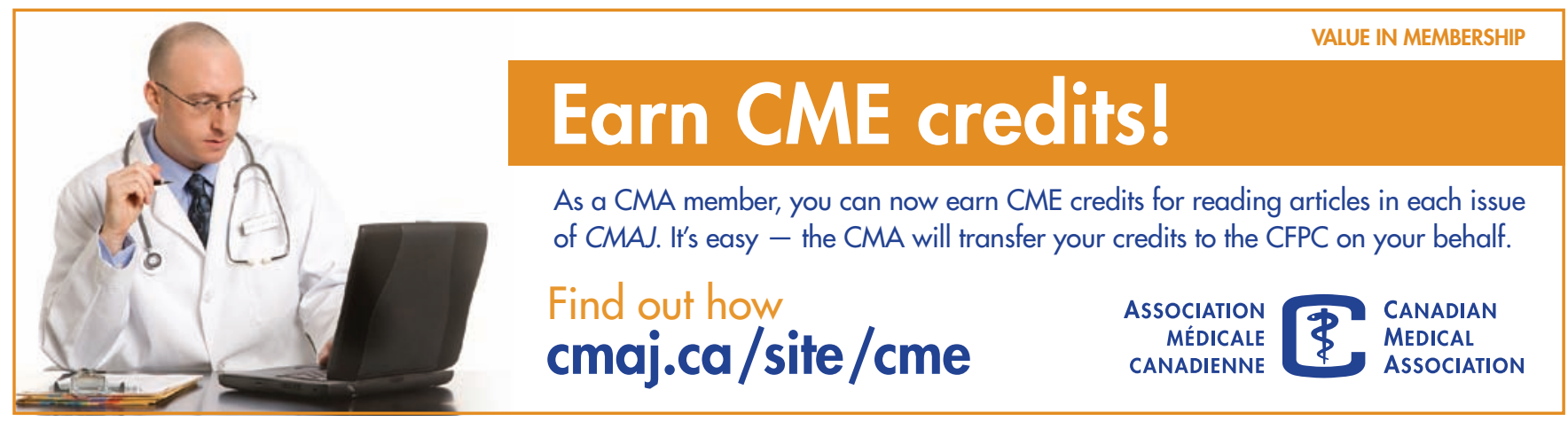

